\title{
Washable, durable and flame retardant conductive textiles based on reduced graphene oxide modification
}

DOI:

10.1007/s10570-019-02884-1

\section{Document Version}

Accepted author manuscript

Link to publication record in Manchester Research Explorer

\section{Citation for published version (APA):}

Zhao, Y., Wang, J., Li, Z., Zhang, X., Tian, M., Zhang, X., Liu, X., Qu, L., \& Zhu, S. (2019). Washable, durable and flame retardant conductive textiles based on reduced graphene oxide modification. Cellulose.

https://doi.org/10.1007/s10570-019-02884-1

\section{Published in:}

Cellulose

\section{Citing this paper}

Please note that where the full-text provided on Manchester Research Explorer is the Author Accepted Manuscript or Proof version this may differ from the final Published version. If citing, it is advised that you check and use the publisher's definitive version.

\section{General rights}

Copyright and moral rights for the publications made accessible in the Research Explorer are retained by the authors and/or other copyright owners and it is a condition of accessing publications that users recognise and abide by the legal requirements associated with these rights.

\section{Takedown policy}

If you believe that this document breaches copyright please refer to the University of Manchester's Takedown Procedures [http://man.ac.uk/04Y6Bo] or contact uml.scholarlycommunications@manchester.ac.uk providing relevant details, so we can investigate your claim.

\section{OPEN ACCESS}


1 Washable, Durable and Flame Retardant Conductive Textiles

2 Based on Reduced Graphene Oxide Modification

3 Yintao Zhao, ${ }^{\text {a,b,c }}$ Jin Wang, ${ }^{\text {a,b,c }}$ Zengqing Li, ${ }^{\text {a,b,c }}$ Xiangwu Zhang, ${ }^{\text {e }}$ Mingwei Tian, ${ }^{\text {a,b,c }}$

4 Xiansheng Zhang, ${ }^{\mathrm{a}, \mathrm{b}, \mathrm{c}}$ Xuqing Liu, ${ }^{\mathrm{d} *}$, Lijun Qu, ${ }^{\mathrm{a}, \mathrm{b}, \mathrm{c} *}$ Shifeng $\mathrm{Zhu}^{\mathrm{a}, \mathrm{b}, \mathrm{c} *}$

$5 \quad{ }^{a}$ College of Textiles and Clothing, Qingdao University, Qingdao, Shandong, 266071, China

$6 \quad{ }^{\mathrm{b}}$ Research Center for Intelligent and Wearable Technology, Qingdao University, Qingdao,

7 Shandong, 266071, China

$8{ }^{\mathrm{c}}$ State Key Laboratory of Bio-Fibers and Eco-Textiles, Qingdao University, Qingdao,

9 Shandong, 266071, China

${ }^{\mathrm{d}}$ The University of Manchester, School of Materia 1s, Manchester, M13 9PL, UK

${ }^{\mathrm{e}}$ Wilson College of Textiles, North Carolina State University, Raleigh, NC 27695, USA

Keywords Polyester/cotton fabric; Graphene oxide; Phosphate flame retardant; Electrical surface resistivity; Flame retardancy

\begin{abstract}
Graphene has been highlighted in a variety of wearable electronics and smart textiles applications due to its unique properties such as high conductivity, transparency, flexibility and other excellent mechanical performance. Although there have been extensive efforts for graphene based conductive fibers/yarns, there are remaining challenges in terms of the seamless integration between $2 \mathrm{D}$ flakes, and reduced charge transport in a lower carrier concentration. Unstable resistance probably arises from the creation of gaps in the conductive parts of the smart textile.
\end{abstract}


Also, regional temperatures can get too high, constituting a fire-safety hazard and endangering the wearer's safety. In this work, the synergistic effect of graphene and flame-retardant materials was investigated, and a conductive fabric was developed which is highly conductive and flame retardancy. Graphene has excellent electrical and thermal conductivity and acts synergistically with traditional flame-retardants on common fabrics. The electrical surface resistivity of hybrid material modified fabrics was as low as $0.54 \mathrm{k} \Omega / \mathrm{sq}$, so they could serve as safe and highly conductive conductor in a simple circuit and show excellent wash-ability. The limiting oxygen index of the fabric increased from 19 to 32 after modification in conjunction with the residue at $800{ }^{\circ} \mathrm{C}$ increased from $17.9 \%$ to $31 \%$, which could be used as safe and highly conductive materials for smart textiles and wearable devices.

\section{Introduction}

Smart textiles have increased exponentially in recent years, being used in many applications such as health, sport, automotive, aerospace and military (Grancarić et al. 2018; Gu et al. 2010). To function as smart textiles, electrical conductivity is a fundamental essential (Zahid et al. 2017; Qing et al. 2019; Cataldi et al. 2017). Many studies have been carried out to fabricate conductive textiles according to these demands and demonstrated in various applications (Simorangkir et al. 2018; Atalay et al. 2018; Zhang and Shi 2019; Huang et al. 2016). Unfortunately, the risking of combustion of electrically conductive fabrics in case of unstable resistance was neglected. For functional textiles, such as fireman garments, various flame retardants have been used to improve the flame retardancy and thermal stability of textile materials such as cotton, polyester/cotton, silk and wool fabrics (Chen et al. 2019; Pan 
et al. 2018; Gashti et al. 2013a; Gashti and Gashti. 2013; Gashti et al. 2012). However, the flame-retardant treatment of the wearable electronics and smart textiles appears rarely in the literature. The aim of the present work was to impart electrical conductivity and flame retardancy to polyester/cotton fabric which is the most widely used fabric material for apparel and technical applications.

Graphene oxide $(\mathrm{GO})$ is a graphene derivative with many polar groups that can easily bind with fiber surfaces (Qu et al. 2019), and it is hydrophilic and can disperse in water readily (Saxena et al. 2011; Cai et al. 2017). Various reduction methods were developed to restore its excellent thermal conductivity, electrical conductivity, and mechanical performance (Gashti and Almasian (2013); Fathy et al. 2019; Nooralian et al. 2016; Voiry et al. 2016). In recent years, many kinds of conductive fabrics have been prepared using reduced graphene oxide (Ding et al. 2019). A cotton fabric with electrical conductivity was fabricated by the deposition of graphene oxide dispersion and hot press reduction, the sheet resistance of modified cotton fabric reduced to 0.9 $\mathrm{k} \Omega / \mathrm{sq}$ (Ren et al. 2017). Nylon-6 yarns, cotton yarns, polyester yarns, and nonwoven fabrics with conductivity were prepared successfully with the addition of graphene oxide and bovine serum albumin (Yun et al. 2013). Additionally, graphene oxide together with other substances has been employed to improve the flame retardancy of materials (Tian et al. 2016; Liu et al. 2019; Feng et al. 2019). The flame retardancy of polypropylene nanocomposites was improved using graphene/multi-walled carbon nanotubes (Huang et al. 2014). The intumescent flame retardant poly(butylene succinate) composites with good flame retardancy were prepared with the application 
of graphene (Wang et al. 2011). Thermal stability and flame retardancy of epoxy resin were significantly improved with the addition of graphene nanosheets (Liu et al. 2014).

In this study, graphene oxide and phosphorus flame retardant were chosen to modify polyester/cotton fabric using the conventional dip-immersion method. The structure, electrical resistance, flammability and thermal property of the fabric have been investigated, and a universal, safe, highly conductive textile material was provided to be used in smart textiles and wearable devices.

\section{Materials and Methods}

Materials

Polyester/cotton fabric (PET/CO65/35, $238 \mathrm{~g} / \mathrm{m}^{2}$ ) was purchased from Qingdao Xu Teng Textile Co., Ltd. Phosphate flame retardant (PFR) was provided by Qingdao Lianmei chemical industry. Aqueous solution of GO (5 g/L) was prepared using flake graphite powder according to modified Hummers' method (Zhao et al. 2018). Large flake graphite powder was kindly provided by Qingdao Huatai Tech Co., Ltd. Hydrochloric acid (37\%), sulfuric acid (98\%), and other reagents were purchased from Tianjin Chemical Reagent Co. Ltd. The reducing agent powder was provided by Ji'nan Tian Shuo Chemical Co., Ltd. The adhesive Goon was provided by Jiahong organic silicon technology Co., Ltd. The distilled water was used in the experiment.

Preparation of modified polyester/cotton fabric

The conventional dip-immersion method was used to modify polyester/cotton fabric. The original fabric (PET/CO), the fabric with reduced graphene oxide (PET/CO-rGO), and the fabric treated with flame retardant (PET/CO-PFR) were chosen as the control samples. The preparation route of the modified fabric (PET/CO-rGO-PFR) was 
shown in Fig. 1. The fabric was firstly soaked in graphene oxide solution for $2 \mathrm{~h}$ to become yellowish-brown and the fabric was squeezed with a small rolling mill to dry at room temperature. Secondly, the fabric was reduced at the temperature of $90{ }^{\circ} \mathrm{C}$ for $1.5 \mathrm{~h}$ and the color of the fabric changed to black, which was caused by the reduction of graphene oxide. This step could be repeated many times to obtain better electrical conductivity. Finally, the sample was dipped and pressed twice in phosphate flame retardant solution, and dried at $90{ }^{\circ} \mathrm{C}$ for 10 mins, $150{ }^{\circ} \mathrm{C}$ for 3 mins. To improve the durability of the modified fabric, the sample was treated in adhesive solution (Goon, $10 \mathrm{wt} \%$ ) for $30 \mathrm{mins}$ and dried at $100^{\circ} \mathrm{C}$ for 15 mins.

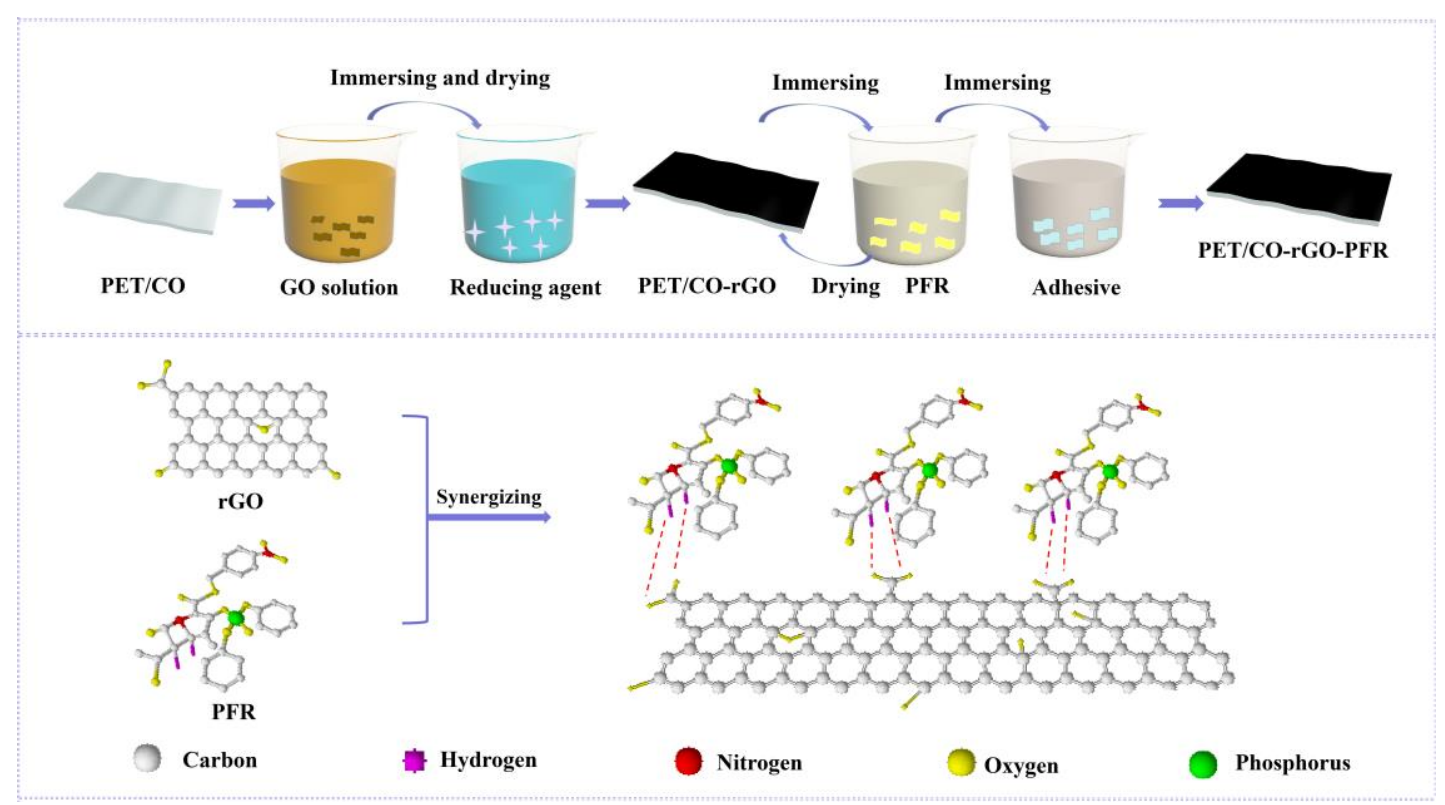

Fig. 1 Preparation route of PET/CO-rGO-PFR fabric

Characterization and measurement

Raman (DXR2, Thermo Scientific, America) spectra were utilized to analyze the G and $\mathrm{D}$ peaks of graphene oxide and reduced graphene oxide on the modified fabric. Fourier transform infrared (FTIR) spectra of all samples were obtained by NICOLET iS5 (Thermo Scientific, America) within the wavelength 500-4000 $\mathrm{cm}^{-1}(\mathrm{KBr}$ disk). The microstructure of samples was measured by scanning electron microscope (SEM, 
EVO18, ZEISS, Germany). Four-point probe technique (RTS-8, Probes Tech, China) was utilized to measure the electrical conductivity, the resistances of ten different positions were gauged to obtain the average value.

The limiting oxygen index (LOI) was tested according to ASTMD2863-08 standard, 10 specimens were prepared for each sample with the size of $5 * 15 \mathrm{~cm}$. Burning behaviors of samples were measured by vertical flame test (VFT) based on GB/T5455-1997 with the size of $5^{*} 12 \mathrm{~cm}$. The thermo gravimetric analysis (TGA, TG209F3, Tarsus, German) was ranged from $50{ }^{\circ} \mathrm{C}$ to $800{ }^{\circ} \mathrm{C}$ with the heating rate 10 ${ }^{\circ} \mathrm{C} / \mathrm{min}$.

\section{Results and discussion}

Raman spectroscopy and Fourier transform infrared spectroscopy

Raman spectra were utilized to analyze the peaks on PET/CO-GO and PET/CO-rGO. As shown in Fig. 2a, G peak at $1590 \mathrm{~cm}^{-1}$ was higher than D peak at $1310 \mathrm{~cm}^{-1}$ in PET/CO-GO. But the phenomenon is quite different in Fig. 2b, D peak occupied the dominant position and the intensity of D peak was higher than G peak with the ratio of ID/IG was 1.23. It can be attributed to the diminishment of $\mathrm{sp}^{2}$ in average size during reduction process. New graphitic domains established and their increase in number proved the well reduction of GO (Stankovich et al. 2007).

The characteristic peaks of rGO, PFR, PET/CO, PET/CO-rGO, PET/CO-PFR and PET/CO-rGO-PFR were listed in Fig. 2c-d. The peaks of PET/CO appeared at 1709 $\mathrm{cm}^{-1}, 1238 \mathrm{~cm}^{-1}, 1104 \mathrm{~cm}^{-1}, 1025 \mathrm{~cm}^{-1}$ were corresponding to stretching vibration of $\mathrm{C}=\mathrm{O}$, asymmetric stretching of aromatic ester, ring asymmetric stretching and stretching vibration of C-O respectively. The peaks between $3600 \mathrm{~cm}^{-1}$ and $3000 \mathrm{~cm}^{-1}$ represented the hydrogen bonded $\mathrm{OH}$ stretching (Carrillo et al. 2004; Alimohammadi et al. 2018; Palaskar et al. 2011). After the final modification, the new peaks appeared 
at $1667 \mathrm{~cm}^{-1}, 1545 \mathrm{~cm}^{-1}, 1420 \mathrm{~cm}^{-1}, 1238 \mathrm{~cm}^{-1}, 1025 \mathrm{~cm}^{-1}, 827 \mathrm{~cm}^{-1}$ corresponding to the bond stretch of $\mathrm{C}=\mathrm{C}$, condensed aromatic ring $\mathrm{O}-\mathrm{H}$, carboxylic $\mathrm{O}-\mathrm{H}, \mathrm{P}-\mathrm{O}-\mathrm{C}$ aromatic stretching, $\mathrm{C}-\mathrm{OH}$ stretching and $\mathrm{P}-\mathrm{CH}_{2}$ bond, respectively (Carrillo et al. 2004; Palaskar et al. 2011; Gaan and Sun 2007; Gashti et al. 2013a; Ren et al. 2007; Gashti et al. 2013b; Shin et al. 1997). It indicated that the flame retardant and rGO were coated on the surface of the fabrics successfully.
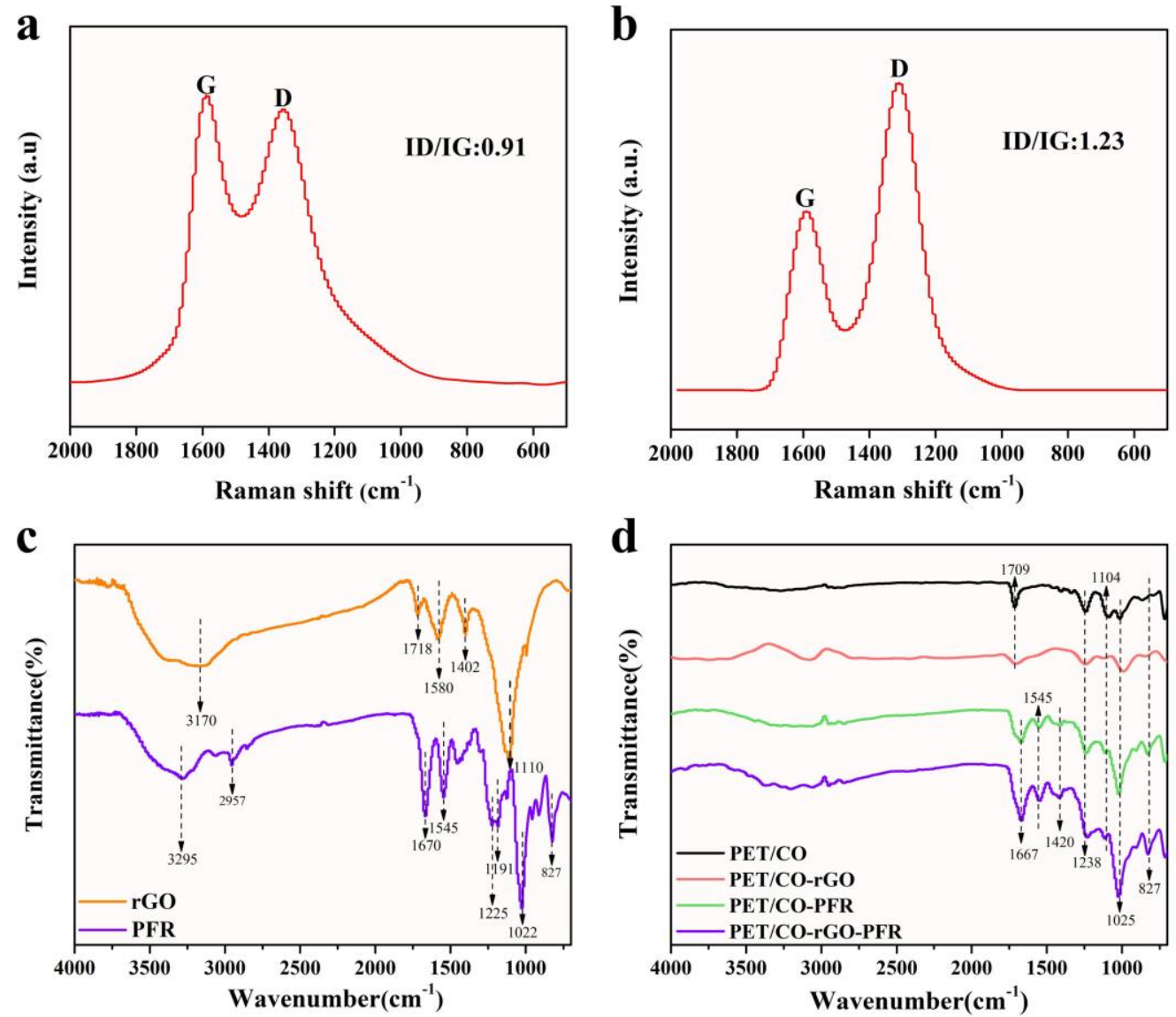

Fig. 2 Raman spectrum of (a) PET/CO-GO and (b) PET/CO-rGO, and FTIR spectra of (c) rGO and PFR, (d) PET/CO, PET/CO-rGO, PET/CO-PFR and PET/CO-rGO-PFR Morphology of polyester/cotton fabric

SEM was used to investigate the micromorphology of samples and the images are illustrated in Fig. 3. The surface of PET/CO was smooth without any other substances seen from Fig. 3a1-a2. After modified by rGO, a thin film could be observed vaguely 
147 on the surface of the fibers (Fig. 3b1-b2). Being treated by PFR, the surface of fibers 148 was covered with PFR as shown in Fig. 3c1-c2. When reduced graphene oxide and 149 PFR were both introduced to the fabric, the continuous and integrated film was 150 formed on the surface of the fibers showing the effective combination of PFR and 151 rGO (Fig. 3d1-d2). 

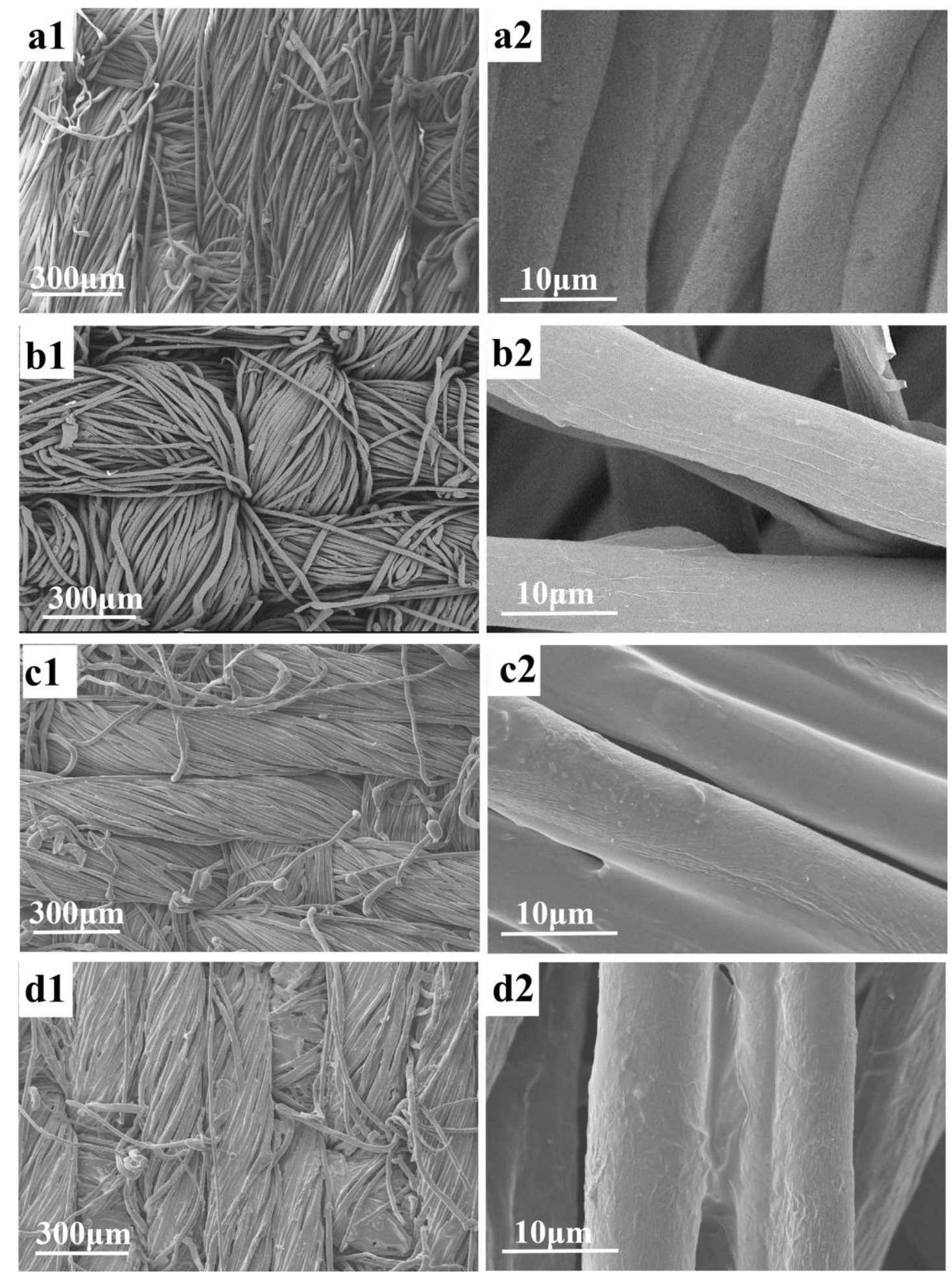

Fig. 3 SEM images of (a1, a2) PET/CO, (b1,b2) PET/CO-rGO, (c1,c2) PET/CO-PFR, (d1,d2) PET/CO-rGO-PFR

155 Electrical surface resistivity

156 As we all know, polyester/cotton fabric was non-conductive. After once modification, 157 PET/CO-rGO and PET/CO-rGO-PFR showed small electrical surface resistivity 
$2.104 \mathrm{k} \Omega / \mathrm{sq}$ and $2.117 \mathrm{k} \Omega / \mathrm{sq}$ respectively. With the increase of reduction times, the electrical surface resistivity decreased furtherly. After being reduced five times, the electrical surface resistivity of fabric reached $0.551 \mathrm{k} \Omega / \mathrm{sq}$ and it increased to 0.559 $\mathrm{k} \Omega / \mathrm{sq}$ after adding PFR which indicated that the existence of PFR showed on negative effect on the electrical conductivity. To show the practical application, light bars and button batteries were connected by PET/CO-rGO-PFR on T-shirt (Fig. 4a), the connecting mode of circuit was exhibited in the left bottom of Fig. 4a. When the circuit was switched on, the light bar lightened up.
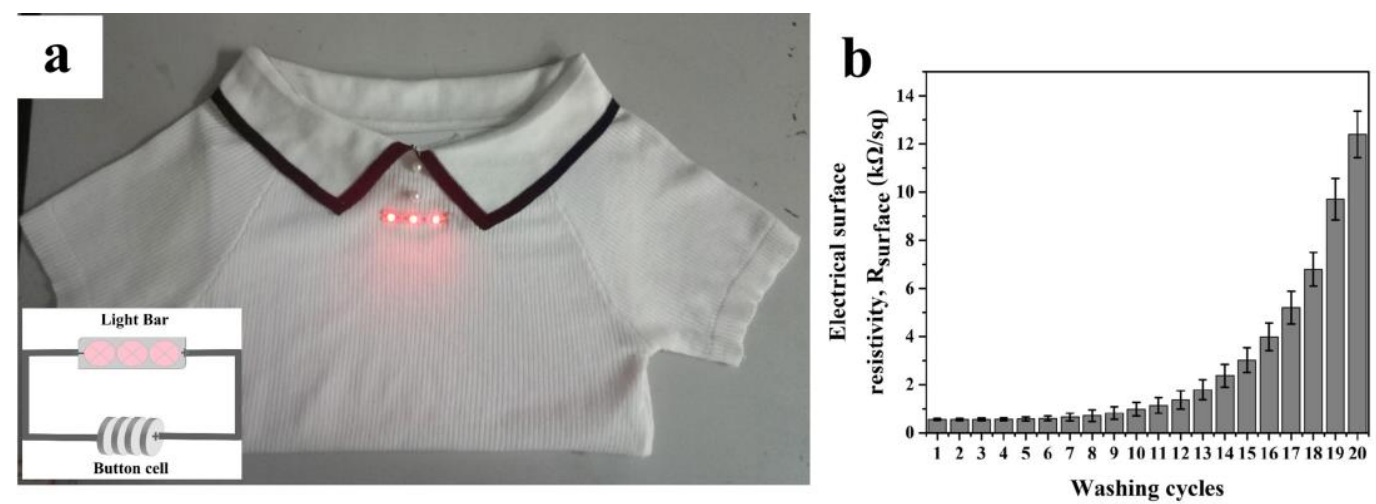

Fig. 4 (a) T-shirts with circuits containing PET/CO-rGO-PFR, and the little picture in (a) is a circuit connected by PET/CO-rGO-PFR (b) Correlation between electrical surface resistivity and washing cycles

To improve the washing fastness of PET/CO-rGO-PFR, the adhesive was used after reduction and the electrical surface resistivity changed a little to $0.546 \mathrm{k} \Omega / \mathrm{sq}$. The relationship between the electrical surface resistivity and washing cycles was illustrated in Fig. 4b indicating that PET/CO-rGO-PFR had good wash resistance with the existence of adhesive.

\section{Flame retardant property}

The images of different samples after Vertical flame test (VFT) were exhibited in Fig. 5a-d. The results showed that PET/CO-PFR and PET/CO-rGO-PFR extinguished themselves, while PET/CO and PET/CO-rGO kept burning after ignition. The 
introduction of rGO decreased the damage length from $3.7 \mathrm{~cm}$ to $3.3 \mathrm{~cm}$ compared with PET/CO-PFR. LOI test was employed to investigate the flame-retardant property of different samples. LOI values of PET/CO, PET/CO-rGO, PET/CO-PFR and PET/CO-rGO-PFR were 19, 20.6, 31, 32, respectively, which indicated that rGO had a synergetic effect on the flame retardancy.

The samples were exerted in the circuit for a long time as shown in Fig. 5e-h. There were smoke and spark of the electrically conductive fabric PET/CO-rGO. After treated by PFR, the circuit operated safely under the same conditions to avoid the combustion.

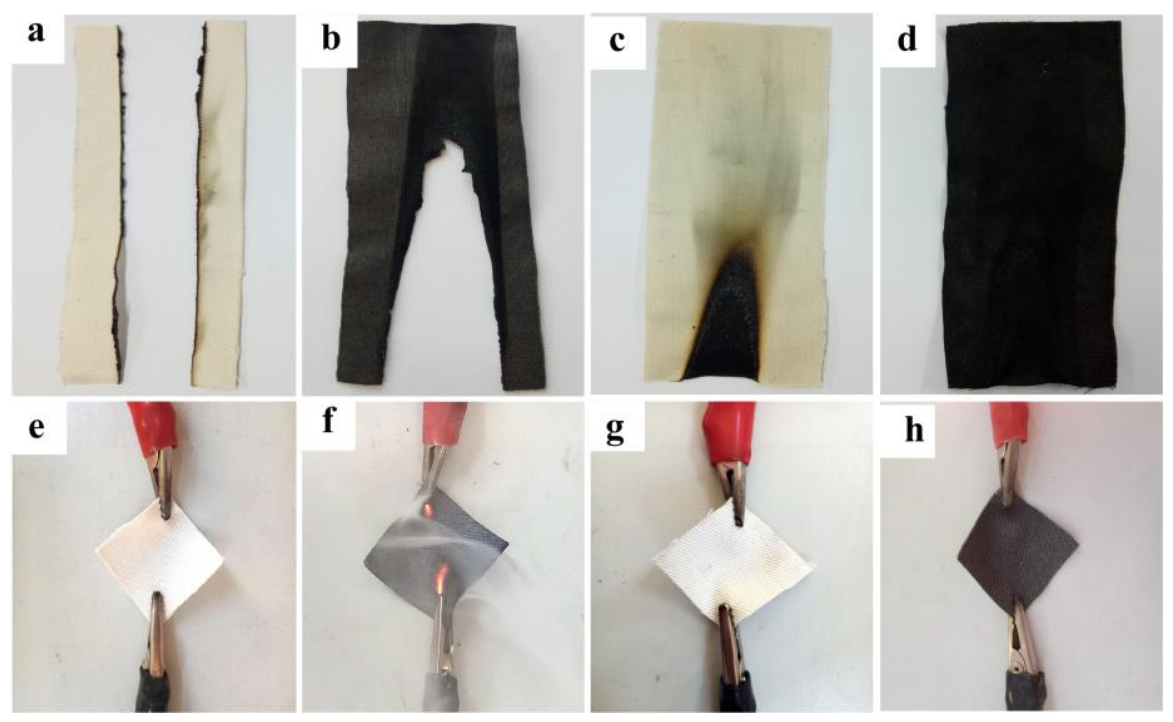

Fig. 5 Images of the samples after VFT (a) PET/CO, (b) PET/CO-rGO, (c) PET/CO-PFR, (d) PET/CO-rGO-PFR, and under voltage connecting (e) PET/CO, (f) PET/CO-rGO, (g) PET/CO-PFR, (h) PET/CO-rGO-PFR

Thermal property

Thermal decomposing behaviors of four samples were evaluated by thermo gravimetric analysis (TGA). TG and DTG curves were shown in Fig. 6a and Fig. 6b. Fig. 6c and Fig. 6d were bar graphs of key data in accordance with TG and DTG curves.

Observing from Fig. 6a and Fig. 6c, the initial decomposition temperature of 
untreated PET/CO fabric was $274{ }^{\circ} \mathrm{C}$ while the temperatures of PET/CO-PFR and PET/CO-rGO-PFR were both $127^{\circ} \mathrm{C}$. The existence of PFR had great effect on the initial decomposition temperature and the addition of rGO did not change the result. The existence of rGO promoted the charring of the fabric and the residues of samples at $800^{\circ} \mathrm{C}$ were $17.9 \%, 30.7 \%, 31 \%$, respectively.

As shown in Fig. 6b, there were two peaks in the DTG curves. The former corresponded to cotton while the latter to polyester (Rahimi et al. 2011). Fig. 6d gave the specific data about DTG curve, the former peaks of the sample treated by PFR and the sample treated by PFR/rGO appeared at $290{ }^{\circ} \mathrm{C}$, which were lower than the original fabric at $355{ }^{\circ} \mathrm{C}$. But the decomposition rate slowed down from $0.37 \% /{ }^{\circ} \mathrm{C}$ to $0.23 \% /{ }^{\circ} \mathrm{C}$. As for the second peaks, it slowed down from $1.51 \% /{ }^{\circ} \mathrm{C}$ to $1.33 \% /{ }^{\circ} \mathrm{C}$. It indicated that PFR lowered the weight loss rate and the addition of rGO promoted it furtherly. It can be interpreted as that phosphorus had a positive effect on flame retardancy of cellulose fibers including cotton and promoted the formation of carbon (El-Shafei et al. 2015). On the other hand, the reduced graphene oxide affected the flame retardancy of polyester for the char formation (Palaskar et al. 2011; Huang et al. 2012). 

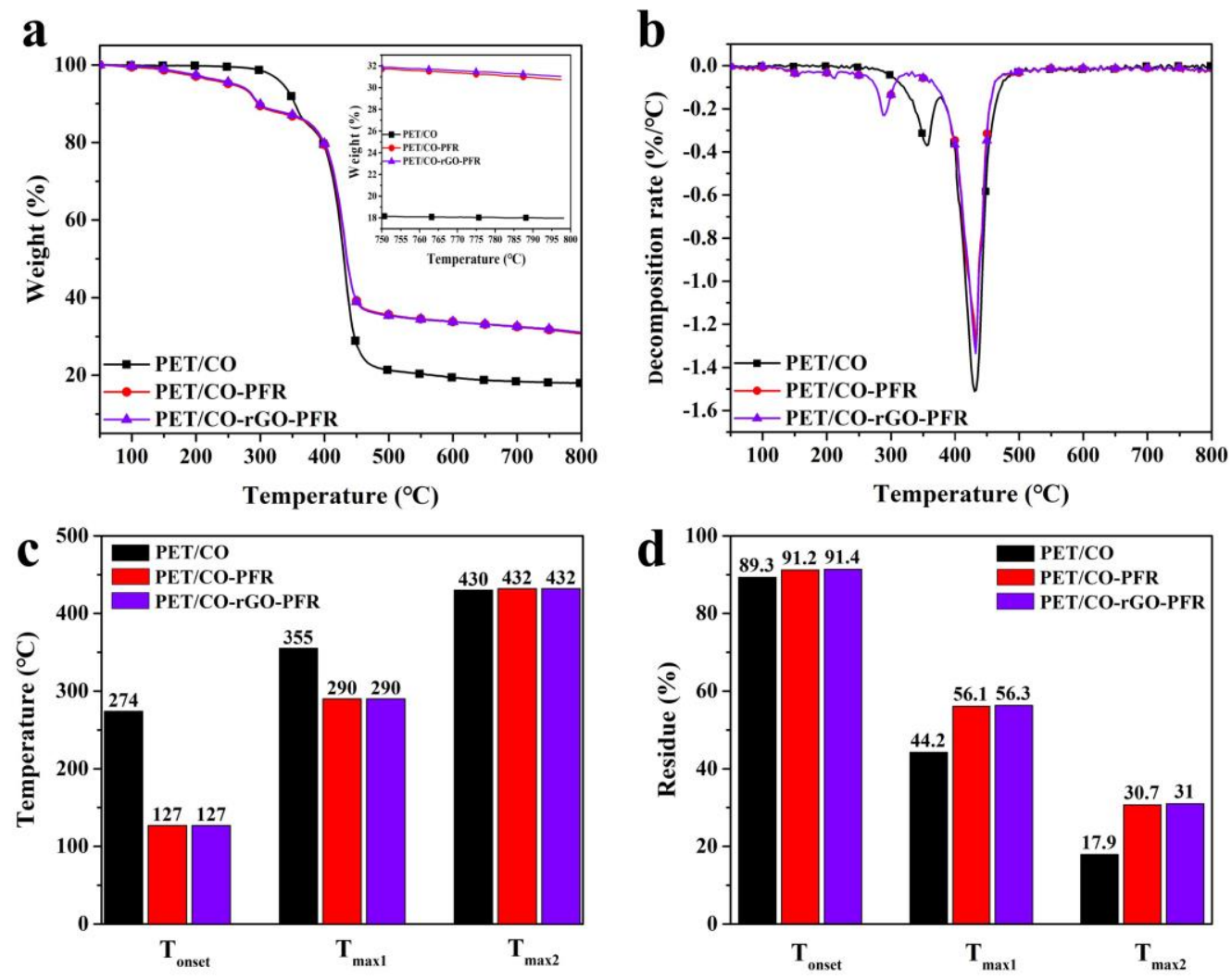

Fig. 6 (a) TGA and (b) DTG curves of PET/CO, PET/CO-PFR, PET/CO-rGO-PFR, the corresponding data of (c) DTG and (d) TG

\section{Conclusions}

In order to develop a non-ignitable electrically conductive fabrics, reduced graphene oxide and phosphate flame retardant were used to modify polyester/cotton fabric. There was cooperative effect between reduced graphene oxide and the flame retardant. Low electrical surface resistivity as $0.54 \mathrm{k} \Omega / \mathrm{sq}$ was achieved, and the introduction of flame-retardant and adhesive had little effect on the surface resistivity. Also, the modified polyester/cotton fabric had good fastness to washing. The existence of rGO improved the flame retardancy compared with the fabric using PFR only. The value of 
entirely. TG and DTG curves showed that thermal stability of fabric improved significantly with a decrease in the maximum mass lose rate.

Acknowledgements The authors would like to acknowledge the financial assistance provided by National Key R\&D Program of China (No 2017YFB0309000) and National Natural Science Foundation of China (No 51672141).

\section{Conflicts of Interest}

The authors declare no conflicts of interest.

\section{References}

Alimohammadi F, Gashti M P, Mozaffari A (2018) Polyvinylpyrrolidone/Carbon Nanotube/Cotton Functional Nanocomposite: Preparation and Characterization of Properties. Fiber. Polym 19: 1940-1947.

Atalay O, Atalay A, Gafford J, Walsh C (2018) A Highly sensitive capacitive-based soft pressure sensor based on a conductive fabric and a microporous dielectric layer. Adv. Mater. Technol 3:1700237-1700244.

Cai G, Xu Z, Yang M, Tang B, Wang X (2017) Functionalization of cotton fabrics through thermal reduction of graphene oxide. Appl Surf Sci 393: 441-448.

Cataldi P, Ceseracciu L, Athanassiou A, Bayer IS (2017) Healable cotton-graphene nanocomposite conductor for wearable electronics. ACS Appl. Mater. Interfaces 9:13825-13830.

Carrillo F, Colom X, Sunol JJ, Saurina J. (2004) Structural FTIR analysis and thermal characterisation of lyocell and viscose-type fibres. Eur. Polym. J. 40: 2229-2234.

Chen T, Hong J, Peng C, Chen G, Yuan C, Xu Y, Dai L (2019) Superhydrophobic and 
flame-retardant cotton modified with DOPO and fluorine-silicon-containing crosslinked polymer. Carbohydr. Polym 208: 14-21.

Ding X, Lei S, Du C, Xie Z, Li J, Huang X (2019) Small-Sized CuS Nanoparticles/N, S Co-Doped rGO Composites as the Anode Materials for High-Performance Lithium-Ion Batteries. Adv. Mater. Interfaces 6:1900038.

El-Shafei A, ElShemy M, Abou-Okeil A (2015) Eco-friendly finishing agent for cotton fabrics to improve flame retardant and antibacterial properties. Carbohydr. Polym 118:83-90.

Fathy M, Moghny TA, Mousa MA (2019) Fast and Fully Scalable Synthesis of Graphene Oxide from Cellulose by Catalytic Acid Spray Method. Arabian J. Sci. Eng 44:305-313.

Feng Y, Li X, Zhao X, Ye Y, Zhou X, Liu H, Xie X (2019) Synergetic improvement in thermal conductivity and flame retardancy of epoxy/silver nanowires composites by incorporating "Branch-Like" flame-retardant functionalized graphene. ACS Appl. Mater. Interfaces 10:21628-21641.

Gaan S, Sun G (2007) Effect of phosphorus and nitrogen on flame retardant cellulose: a study of phosphorus compounds. J. Anal. Appl. Pyrolysis 78:371-377.

Gashti MP, Navid MY, Rahimi MH (2012) Coating of macroemulsion and microemulsion silicones on poly (ethylene terephthalate) fibers: Evaluation of the thermal properties and flammability. J. Appl. Polym. Sci 125:1430-1438.

Gashti MP, Navid MY, Rahimi MH (2013) Effects of coating of nano-and microemulsion silicones on thermal properties and flammability of polyethylene terephthalate textile. Pigm. Resin Technol 42:34-44.

Gashti MP, Gashti MP (2013) Effect of colloidal dispersion of clay on some properties 
Gashti MP, Pournaserani A, Ehsani H, Gashti, MP (2013a) Surface oxidation of cellulose by ozone-gas in a vacuum cylinder to improve the functionality of fluoromonomer. Vacuum 91:7-13.

Gashti MP, Elahi A, Gashti MP (2013b) UV radiation inducing succinic acid/silica kaolinite network on cellulose fiber to improve the functionality. Composites Part B 48:158-166.

Gashti MP, Almasian A (2013) Citric acid/ZrO2 nanocomposite inducing thermal barrier and self-cleaning properties on protein fibers. Composites Part B $52: 340-349$.

Grancarić AM, Jerković I, Koncar V, Cochrane C, Kelly FM, Soulat D, Legrand X (2018) Conductive polymers for smart textile applications. J. Ind Text 48:612-642.

Gu JF, Gorgutsa S, Skorobogatiy M (2010) Soft capacitor fibers using conductive polymers for electronic textiles. Smart Mater. Struct 19:1-13.

Huang G, Liu L, Wang R, Zhang J, Sun X, Peng H (2016) Smart color-changing textile with high contrast based on a single-sided conductive fabric. J. Mater. Chem. C 4:7589-7594.

Huang G, Gao J, Wang X, Liang H, Ge C (2012) How can graphene reduce the flammability of polymer nanocomposites?. Mater. Lett 66:187-189.

Hu G, Zhao C, Zhang S, Yang M, Wang Z (2006) Low percolation thresholds of electrical conductivity and rheology in poly (ethylene terephthalate) through the networks of multi-walled carbon nanotubes. Pol 47:480-488.

Huang G, Wang S, Song P, Wu C, Chen S, Wang X (2014) Combination effect of 
carbon nanotubes with graphene on intumescent flame-retardant polypropylene nanocomposites. Composites. Part A 59:18-25.

Liu S, Yan H, Fang Z, Wang H (2014) Effect of graphene nanosheets on morphology, thermal stability and flame retardancy of epoxy resin. Compos. Sci. Technol 90:40-47.

Liu L, Chen Y, Liu H, Rehman HU, Chen C, Kang H, Li H (2019) A graphene oxide and functionalized carbon nanotube based semi-open cellular network for sound absorption. Soft matter 15:2269-2276.

Nooralian Z, Gashti MP, Ebrahimi I (2016) Fabrication of a multifunctional graphene/polyvinylphosphonic acid/cotton nanocomposite via facile spray layer-by-layer assembly. RSC Adv 6:23288-23299.

Palaskar S, Kale KH, Nadiger GS, Desai AN (2011) Dielectric barrier discharge plasma induced surface modification of polyester/cotton blended fabrics to impart water repellency using HMDSO. J. Appl. Polym. Sci 122:1092-1100.

Pan Y, Liu L, Wang X, Song L, Hu Y (2018) Hypophosphorous acid cross-linked layer-by-layer assembly of green polyelectrolytes on polyester-cotton blend fabrics for durable flame-retardant treatment. Carbohydr Polym 201:1-8.

Qing X, Wang Y, Zhang Y, Ding X, Zhong W, Wang D, Lu Z (2019) Wearable Fiber-Based Organic Electrochemical Transistors as a Platform for Highly Sensitive Dopamine Monitoring. ACS Appl. Mater. Interfaces 11:13105-13113.

Qu J, He N, Patil SV, Wang Y, Banerjee D Gao W (2019) Screen Printing of Graphene Oxide Patterns onto Viscose Nonwovens with Tunable Penetration Depth and Electrical Conductivity. ACS Appl. Mater. Interfaces 11:14944-14951.

Rahimi MH, Parvinzadeh M, Navid MY, Ahmadi S (2011) Thermal characterization 
and flammability of polyester fiber coated with nonionic and cationic softeners. J. Surfactants Deterg 14:595-603.

Ren H, Sun J, Wu B, Zhou Q (2007) Synthesis and properties of a phosphorus-containing flame retardant epoxy resin based on bis-phenoxy (3-hydroxy) phenyl phosphine oxide. Polym. Degrad. Stab 92:956-961.

Ren J, Wang C, Zhang X, Carey T, Chen K, Yin Y, Torrisi F (2017) Environmentally-friendly conductive cotton fabric as flexible strain sensor based on hot press reduced graphene oxide. Car 111:622-630.

Saxena S, Tyson TA, Shukla S, Negusse E, Chen H, Bai J (2011) Investigation of structural and electronic properties of graphene oxide. Appl. Phys. Lett 99:1838-1845.

Simorangkir RB, Yang Y, Esselle KP, Zeb BA (2018) A. Method to realize robust flexible electronically tunable antennas using polymer-embedded conductive fabric. IEEE Trans. Antennas Propag 66:50-58.

Shin S, Jang J, Yoon SH, Mochida I (1997) A study on the effect of heat treatment on functional groups of pitch based activated carbon fiber using FTIR. Car 35:1739-1743.

Stankovich S, Dikin DA, Piner RD, Kohlhaas KA, Kleinhammes A, Jia Y, Ruoff RS (2007). Synthesis of graphene-based nanosheets via chemical reduction of exfoliated graphite oxide. Car 45: 1558-1565.

Tian M, Hu, X Qu L, Du M, Zhu S, Sun Y, Han, G (2016) Ultraviolet protection cotton fabric achieved via layer-by-layer self-assembly of graphene oxide and chitosan. Appl Surf Sci 377:141-148. 
Voiry D, Yang J, Kupferberg J, Fullon R, Lee C, Jeong HY Chhowalla M (2016) High-quality graphene via microwave reduction of solution-exfoliated graphene oxide, Science 353:1413-1416

Wang X, Song L, Yang H, Lu H, Hu Y (2011) Synergistic effect of graphene on antidripping and fire resistance of intumescent flame-retardant poly (butylene succinate) composites. Ind. Eng. Chem. Res 50:5376-5383.

Yun Y, Hong W, Kim WJ, Jun Y, Kim BH (2013) A novel method for applying reduced graphene oxide directly to electronic textiles from yarns to fabrics. Adv. Mater 25:5701-5705.

Zahid M, Papadopoulou EL, Athanassiou A, Bayer IS (2017) Strain-responsive mercerized conductive cotton fabrics based on PEDOT: PSS/graphene. Mater. Des 135:213-222.

Zhang X, Shi M (2019) Flame Retardant Vinylon/Poly (m-phenylene isophthalamide) Blended Fibers with Synergistic Flame Retardancy for Advanced Fireproof Textiles. J. Hazard. Mater 365:9-15.

Zhao H, Tian M, Hao Y, Qu L, Zhu S, Chen S (2018) Fast and facile graphene oxide grafting on hydrophobic polyamide fabric via electrophoretic deposition route. J. Mater. Sci 53:9504-9520. 